\title{
Meta
}

Journal des traducteurs

Translators' Journal

\section{BARRADA, Samia et Yousif ELIAS (1992) : Traduire le discours économique : implications didactiques pour la traduction français-arabe, Tanger, Publications de l'École Supérieure Roi Fahd de Traduction, 214 p. + 7 pages d'annexes}

\section{Ahmed Elkhamloussy}

Volume 38, numéro 3, septembre 1993

URI : https://id.erudit.org/iderudit/002701ar

DOI : https://doi.org/10.7202/002701ar

Aller au sommaire du numéro

Éditeur(s)

Les Presses de l'Université de Montréal

ISSN

0026-0452 (imprimé)

1492-1421 (numérique)

Découvrir la revue

Citer ce compte rendu

Elkhamloussy, A. (1993). Compte rendu de [BARRADA, Samia et Yousif ELIAS (1992) : Traduire le discours économique : implications didactiques pour la traduction français-arabe, Tanger, Publications de l'École Supérieure Roi Fahd de Traduction, 214 p. +7 pages d'annexes]. Meta, 38(3), 563-564.

https://doi.org/10.7202/002701ar d'utilisation que vous pouvez consulter en ligne. 
BARRADA, Samia et Yousif ELIAS (1992): Traduire le discours économique : implications didactiques pour la traduction français-arabe, Tanger, Publications de l'École Supérieure Roi Fahd de Traduction, 214 p. +7 pages d'annexes.

Samia Barrada et Yousif Elias sont deux professeurs à l'École Supérieure Roi Fahd de Traduction à Tanger, au Maroc. Ils enseignent respectivement le français de spécialité et les techniques de rédaction, et la traduction français-arabe.

Leur manuel se présente comme «un outil de travail et de réflexion aux étudiants en traduction économique et éventuellement à leurs enseignants». Les problèmes de la traduction économique y sont traités de façon systématique. En effet, dans la première partie du livre, les auteurs traitent de l'analyse du discours et de son applicabilité à la traduction économique. Cette partie comprend trois grandes rubriques : a) la compréhension (aspects terminologique, syntaxique, stylistique et pragmatique), b) la communication (objectivation, métaphorisation, métonymisation, non-dit du discours, etc.) et c) le perfectionnement (connaissances linguistiques et compétence rédactionnelle). Cette partie illustre l'influence de la théorie interprétative sur les auteurs.

Soucieux de l'importance de l'adéquation entre la théorie et la pratique, les auteurs consacrent la deuxième partie de leur ouvrage à l'application. Ils suggèrent des méthodes de travail et non des recettes toutes faites. En effet, ils proposent trois textes qui font l'objet d'une analyse présentée sous forme de fiches: fiche (1) organisation textuelle (logique qui sous-tend le texte), fiche (2) identification et résolution de difficultés textuelles et extratextuelles (allusions, métaphores, etc.) et fiche (3) termes et collocations. Ces trois fiches sont suivies d'une traduction intégrale du texte et de notes de traduction qui expliquent le processus traductionnel, proposent des variantes et justifient les choix.

La partie qui succède à ces trois textes comporte un ensemble de textes que les auteurs classent sous deux rubriques: textes de vulgarisation et textes d'un niveau élevé 
de spécialisation. Ces textes sont présentés comme des exercices, à la fois de traduction et de perfectionnement linguistique. Les auteurs proposent ces exercices selon une séquence progressive, par degrés de difficultés.

Les auteurs ont également réuni une série de documents de référence de deux types: documents qui traitent du lexique, de la terminologie, des sigles, etc. et documents portant sur des thèmes particuliers ayant trait à l'économie. À la fin du manuel, ils présentent une bibliographie qui comprend des ouvrages sur la traduction, la terminologie et la langue générale et de spécialité. Toutefois, quelques coquilles se sont discrètement glissées dans le nom de certains auteurs et le titre de certains ouvrages, sans pour autant avoir une incidence sur la valeur pédagogique du manuel.

Pour conclure, je dirais donc que ce manuel comprend tous les éléments qui en font un bon outil de travail et de réflexion. Il est conçu selon les principes de l'enseignement par objectifs d'apprentissage; les difficultés de traduction y sont traitées de façon systématique; il allie la théorie à la pratique; et enfin, il propose des exercices d'application par degrés de difficultés.

AHMED ELKHAMLOUSSY Université d"Ottawa, Ottawa, Canada 